\title{
An Optimized Ensemble Model for Prediction the Bandwidth of Metamaterial Antenna
}

\author{
Abdelhameed Ibrahim ${ }^{1, *}$, Hattan F. Abutarboush ${ }^{2}$, Ali Wagdy Mohamed ${ }^{3,4}$, Mohamad Fouad ${ }^{1}$ and \\ El-Sayed M. El-kenawy ${ }^{5,6}$
}

${ }^{1}$ Computer Engineering and Control Systems Department, Faculty of Engineering, Mansoura University, Mansoura, 35516, Egypt

${ }^{2}$ Electrical Engineering Department, College of Engineering, Taibah University, Medina, 42353, Saudi Arabia

${ }^{3}$ Operations Research Department, Faculty of Graduate Studies for Statistical Research, Cairo University, Giza, 12613, Egypt

${ }^{4}$ Wireless Intelligent Networks Center (WINC), School of Engineering and Applied Sciences, Nile University, Giza, Egypt

${ }^{5}$ Department of Communications and Electronics, Delta Higher Institute of Engineering and Technology, Mansoura, 35111, Egypt

${ }^{6}$ Faculty of Artificial Intelligence, Delta University for Science and Technology, Egypt

*Corresponding Author: Abdelhameed Ibrahim. Email: afai79@mans.edu.eg

Received: 18 July 2021; Accepted: 19 August 2021

\begin{abstract}
Metamaterial Antenna is a special class of antennas that uses metamaterial to enhance their performance. Antenna size affects the quality factor and the radiation loss of the antenna. Metamaterial antennas can overcome the limitation of bandwidth for small antennas. Machine learning (ML) model is recently applied to predict antenna parameters. ML can be used as an alternative approach to the trial-and-error process of finding proper parameters of the simulated antenna. The accuracy of the prediction depends mainly on the selected model. Ensemble models combine two or more base models to produce a better-enhanced model. In this paper, a weighted average ensemble model is proposed to predict the bandwidth of the Metamaterial Antenna. Two base models are used namely: Multilayer Perceptron (MLP) and Support Vector Machines (SVM). To calculate the weights for each model, an optimization algorithm is used to find the optimal weights of the ensemble. Dynamic Group-Based Cooperative Optimizer (DGCO) is employed to search for optimal weight for the base models. The proposed model is compared with three based models and the average ensemble model. The results show that the proposed model is better than other models and can predict antenna bandwidth efficiently.
\end{abstract}

Keywords: Metamaterial antenna; machine learning; ensemble model; multilayer perceptron; support vector machines

\section{Introduction}

Metamaterial antenna is extensively reported in the literature because of its unusual properties [1]. Metamaterials are artificially engineered materials to add extra properties that do not 
exist in the original materials [2]. They derive their extraordinary properties not from the base material, but the internal structure after manipulating their internal structure. The new properties may enhance the way the material manipulates electromagnetic waves or change its permittivity index. These properties improve the capabilities of the original material and their engagement in the industry.

To design an antenna, the dimension of the antenna can be calculated by researchers by using a mathematical formula. Then a simulator can be employed to find the parameters such as bandwidth. In case that the parameters don't meet the expectation, the simulated antenna's dimension should be adjusted. This is repeated until the desired parameters are reached. Researchers are doing this process usually by trial-and-error method and this process can take long time [3,4].

Metamaterials are widely used in various applications such as Metamaterial absorber [5], Metamaterial Lens [6], Wireless Power Transfer [7], and Ultrasensitive sensor [8]. Because of their wide range of applications, Metamaterials have received a huge research interest in different sciences. Applications of Metamaterial also include Metamaterial Antenna [9] which is a special class of antennas that uses metamaterial to enhance their performance. An antenna is used to propagate energy into free space. It is a fact that antenna size affects the quality factor and the radiation loss of the antenna [10]. However, a small size antenna that has low cost and good efficiency is preferred for the integrated antenna. The metamaterial can improve the performance of small antennas as it could improve their bandwidth and gain. Moreover, it can reduce their electrical size and it can improve their directivity. Metamaterial antennas can overcome the limitation of bandwidth for small antennas.

To estimate the effect of metamaterial, simulation software is used [11]. CST Microwave Studio is an example of electromagnetic simulation software that can simulate complex structures. After the simulation process, antenna parameters can be estimated such as bandwidth, gain, Voltage Standing Wave Ration (VSWR), and return loss. During the simulation process, researchers may use trial and error to adjust Metamaterial Antenna to meet the expectation of antenna parameters. This process could take an unpredicted long time. The alternative approach is to use a Machine learning model to predict antenna parameters [12].

Machine Learning (ML) is a very popular research topic that has been extensively used in several applications in literature [13]. ML is a subfield of artificial intelligence that cares about building models based on data to make decisions or predictions. These models are not explicitly programmed to do so but they learn from training data. Examples of applications of ML are Computer Vision [14], Forecasting Solar Radiation [15], Disease Classification [16] and Robotics [17].

In Metamaterial simulation, ML can be used as an alternative approach to the trial-anderror process of finding proper parameters of the simulated antenna. ML model can be a quick prediction if it has been trained using a dataset. The accuracy of the prediction depends mainly on the selected model. Many machine learning models are reported in the literature such as Artificial Neural Networks (ANN) [18], Support Vector Machines (SVM) [19], Decision Tree (DT) [20], and K-Nearest Neighbor (KNN) [21]. In Metamaterial Antenna, researchers have used these models to predict different parameters of Antenna. Ensemble models combine two or more base models to produce a better-enhanced model. There are some methods for combing base models such as average ensemble, boosting, bagging, and weighted average ensemble.

In this paper, a weighted average ensemble model is proposed to predict the bandwidth of the Metamaterial Antenna. Two base models are used namely: Multilayer Perceptron (MLP) 
and Support Vector Machines (SVM). To calculate the weights for each model, an optimization algorithm is used to find the optimal weights of the ensemble. In this paper, Dynamic GroupBased Cooperative Optimizer (DGCO) [22] is used to search for optimal weight for the base models. The proposed model is compared with three based models and the average ensemble model. The results show that the proposed model is better than other models and can predict antenna bandwidth efficiently.

This paper is structured as follows: Literature review is presented in Section 2. Data preprocessing and the proposed ensemble model is explained in Section 3. Results are shown and discussed in Section 4. Finally, the conclusion of the presented work is discussed in Section 6.

\section{Literature Review}

Machine learning models have been extended in several areas such as telecommunication [23], Computer Vision [24], Disease Classification [25], Solar Energy [26], and Network Security [27]. These models learn from data. It can classify or predict the target data without being explicitly programmed.

\subsection{Machine Learning Models}

There are many machine learning algorithms such as Artificial Neural Network (ANN) [18], Support Vector Machines (SVM) [19], K-Nearest Neighbor (KNN) [21], and Decision Tree (DT) [20]. These algorithms apply different techniques to build a model that can generalize and predict hidden data that the model did not see in the training phase. For instance, ANN is an intelligent mathematical model that is inspired by the biological nervous system [18]. Multilayer Perceptron (MLP) is one of the most popular architectures of feedforward ANN. MLP consists of one input layer, one or more hidden layers, and one output layer. Fig. 1 depicts the structure of MLP. As shown in the figure, each layer consists of a group of neurons that are connected to the neurons in the next layer using connection weights. These weights are adjusted during the learning process of MLP.

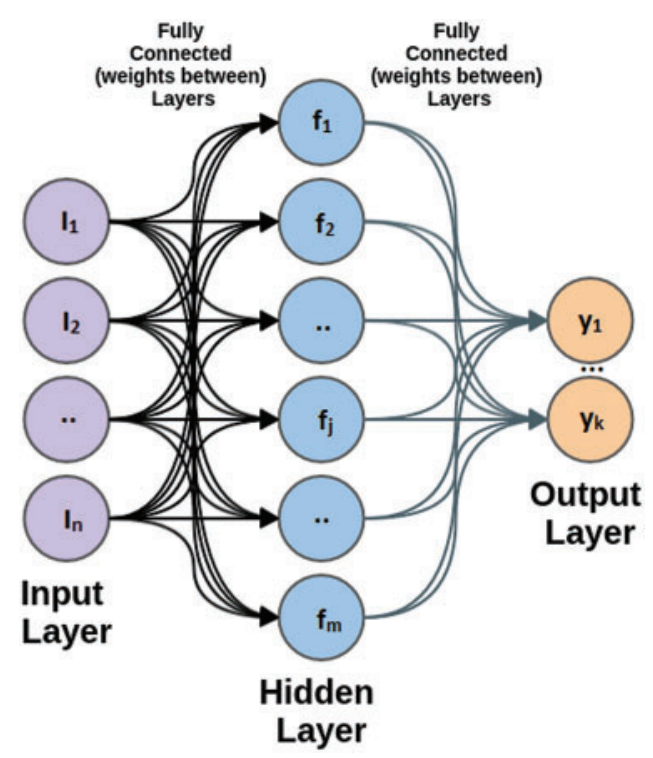

Figure 1: Structure of multilayer perceptron 
ANN has been used in various applications in the literature. For instance, in [28], the authors used ANN to build a smart antenna capable of tracking the angles of arrival of multiple sources. In [29], ANN is used to optimize the dimensions of the microstrip patch antenna. Additionally, in [30], ANN is trained to replace the full-wave analysis to optimize the bandwidth of the dualring antenna. In [31], the authors trained ANN using geometric parameters of the broadband antenna to predict the input impedance.

Support Vector Machines (SVM) [19] is a robust machine learning algorithm that has been highly reported in the literature. It builds a model by finding the optimal hyperplane that can maximize the margin and minimize the error [32]. Recently, SVM has been used in various tasks of communications. In [33], SVM has been used with multiple antennas in multiuser communication systems. The authors proposed an antenna allocation system based on SVM. This allocation system reduced computation complexity in the online antenna. In [34], the authors used data collected from a microwave simulator to train SVM to design the feed section of a microstrip patch antenna.

\subsection{Machine Learning Ensemble}

In literature, researchers combine two or model machine learning models to enhance the performance and overcome the disadvantages of single weak learners (base models). These model combinations are called ensemble models [35]. The simple form of the ensemble is an average ensemble that calculates its output using the average of outputs of base models. In the average ensemble, each base model has the same weight and strength in the calculation. This may result in an undesired performance because the average ensemble deals with all base models equally. On the other hand, the weighted average ensemble is the smart version of the average ensemble that gives weight to each model. These weights give importance to good base models and low importance to other models. To calculate these weights efficiently, an optimization algorithm can be used. In the next section, the DGCO optimizer is discussed in detail.

In [36], the authors have used an ensemble model of multiple neural networks to calculate the optimal dimensions of the circular fractal patch antenna. Their model could reduce the size of the fractal antenna by $41.64 \%$ and has been used to design a $3.8 \mathrm{GHz}$ WLAN antenna. In [37], an ensemble of neural networks has been used to minimize the design of the circular fractal antenna. Their model has been used to develop an antenna at $2.45 \mathrm{GHz}$.

\subsection{DGCO Optimizer}

Dynamic Group-Based Cooperative Optimization algorithm (DGCO) [22] is a recently proposed optimization algorithm that is influenced by the cooperative behavior adopted by swarm individuals to achieve their global goals. DGCO is a population-based optimization algorithm that starts the optimization process with initial random individuals. These individuals act as a candidate solution to the optimization problem. Then, DGCO calculates the fitness value for each individual in the population. Based on the fitness values, the leader solution (leader) can be estimated. After that, DGCO divides the population into exploitation group and exploration group. Each group is responsible for a specific task in the optimization process. The exploration group is responsible for discovering and exploring the search space to find promising locations. To perform this task, individuals in this group uses two strategies. The first strategy is to search around the solution. In this strategy, a solution moves randomly to a random location near its current location. This strategy is modeled using the following formulas:

$\vec{D}=r_{1} \cdot(\vec{S}(t)-1)$ 
$\vec{S}(t+1)=\vec{S}(t)+\vec{D} \cdot\left(2 \vec{r}_{2}-1\right)$

where $\overrightarrow{r_{1}}$ and $\overrightarrow{r_{2}}$ are vectors within $[0,2]$ and $[0,1]$ respectively, $t$ represents the current iteration, vector $\vec{S}$ indicates the current solution, and $\vec{D}$ is the distance. The other exploration strategy is a mutation in which the solutions move, in the search space, to a random location. This strategy improves the diversity of the population and helps to avoid local optima stagnation.

On the other hand, the exploitation group is responsible for improving existing solutions by finding more promising locations around them. To achieve that, individuals in the exploration group apply two strategies. The first strategy is to move towards the best solution using random walks. This strategy is modeled as follows:

$\vec{D}=\overrightarrow{r_{3}} \cdot(\vec{L}(t)-\vec{S}(t))$

$\vec{S}(t+1)=\vec{S}(t)+\vec{D}$

where vector $\overrightarrow{r_{3}}$ has a random value within $[0,2]$ which controls the movement to the leader solution and the vector $\vec{L}$ represents the best solution. The other strategy is to search around the best solution. In this strategy, the solution changes its location to a random location near the best solution. This technique is modeled as follows:

$\vec{D}=\vec{L}(t) *\left(\vec{k}-r_{4}\right)$

$\vec{S}(t+1)=\vec{S}(t)+\vec{D} \cdot\left(2 \overrightarrow{r_{5}}-1\right)$

$\vec{k}=2-\frac{2 \times t^{2}}{\text { iters_count }^{2}}$

where vectors $\vec{r}_{4}$ and $\vec{r}_{5}$ have random values within $[0,1], \vec{k}$ has values that are decreasing exponentially from 2 to 0 during iterations and $\vec{D}$ represents the diameter of the circle in which the solution will look for better solutions.

One of the merits of the DGCO algorithm is its ability to perform a good balance between exploration and exploitation. In each iteration, DGCO dynamically changes the number of individuals in each group based on the convergence history. It starts with a higher number of individuals in the exploration group ( $70 \%$ for instance) then over the course of iterations this number decreases and the number of individuals in the exploitation group increases. Based on the convergence history of the last three iterations, DGCO decides whether to increase the number of individuals in the exploration group or note. This balance helps in enhancing the performance of the optimization process as well as avoiding local optima stagnation. Finally, DGOC randomly interchanges the roles of individuals of each group in each iteration to enhance the diversity of the population. The flow chart of the DGCO algorithm is presented in Fig. 2. 


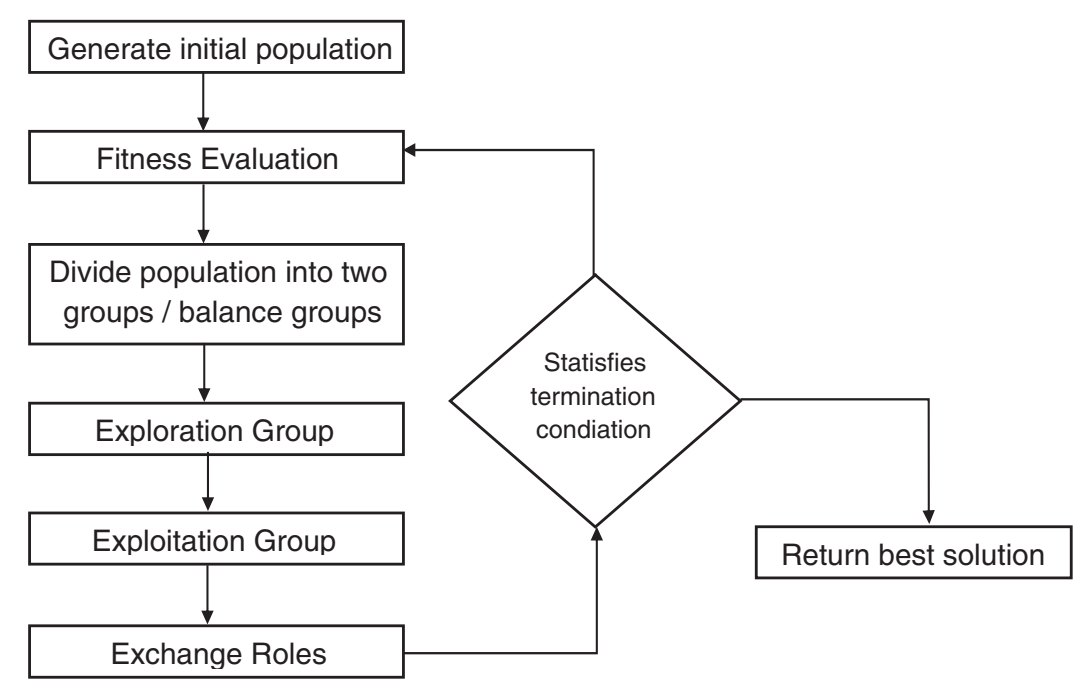

Figure 2: Flow chart of DGCO optimization algorithm

\section{Proposed Ensemble Model}

In this section, the proposed ensemble model will be presented and explained in detail. First, the dataset is presented. An expletory analysis of the dataset is presented. Then, the preprocessing techniques applied to the dataset will be discussed. Second, for the calculation of the weights for base models, the Dynamic Group-Based Cooperative Optimizer (DGCO) algorithm is used to find the optimal weights of the ensemble model as shown in Fig. 3.

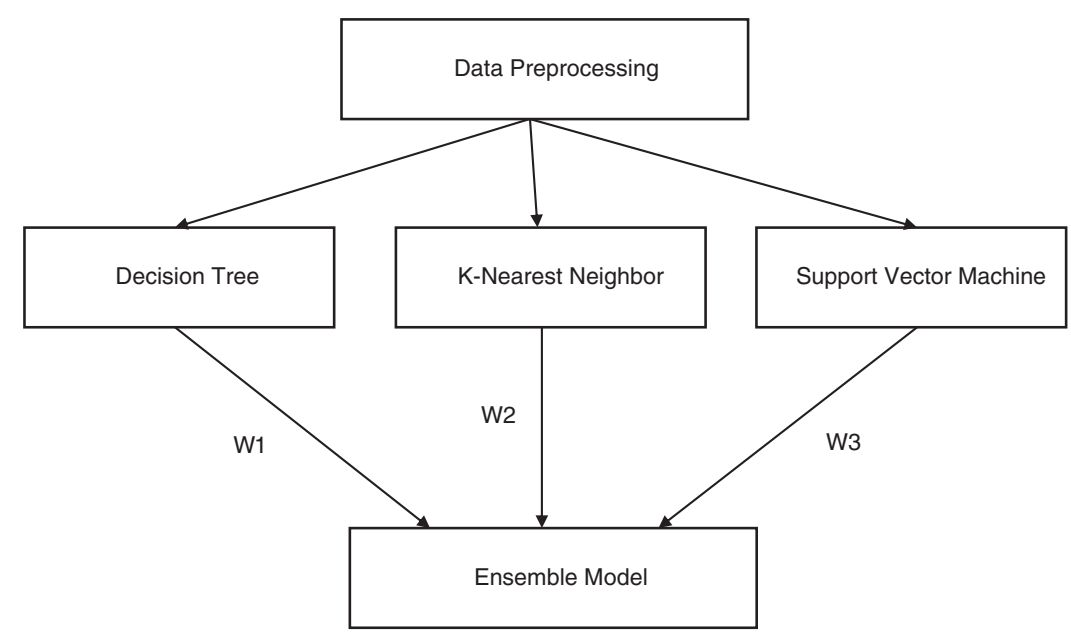

Figure 3: Weight average ensemble model

\subsection{Data Preprocessing}

The dataset used in this work contains 11 features of the Metamaterial Antenna project. The dataset has been downloaded from Kaggle [38]. It contains 572 records. Each record contains parameters of the metamaterial antenna as follows: width and height of split ring resonator, 
the distance between rings, the width of rings, the gap between rings, the distance between antenna patch and array, number of split ring resonator cells in an array, gain of antenna, the distance between split ring resonator cells in the array, the bandwidth of the antenna and return loss, and voltage standing wave ration of the antenna. To control its magnetic susceptibility of metamaterial, Split Ring Resonator (SRR) structure is shown in Fig. 4. The SRR formulation contains two rings which are separated by a gap. The SRR importance in a metamaterial antenna is to enhance the bandwidth and reduce mutual coupling [39]. Tab. 1 describes each feature of the dataset. These features will be used to predict the bandwidth of the antenna using a machine learning model.

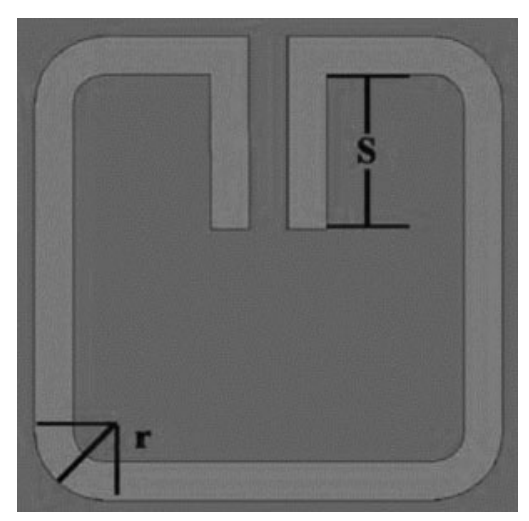

Figure 4: Shape of SRR [39]

Table 1: Description of features of the dataset

\begin{tabular}{lll}
\hline$\#$ & Feature & Description \\
\hline $\mathbf{1}$ & Wm & Split ring resonator's width and height \\
$\mathbf{2}$ & W0m & Gap between rings \\
$\mathbf{3}$ & Dm & Distance between rings \\
$\mathbf{4}$ & Tm & Width of rings \\
$\mathbf{5}$ & SRR_num & \# Split ring resonator cells \\
$\mathbf{6}$ & Xa & Distance between antenna patch and array \\
$\mathbf{7}$ & Ya & Distance between split ring resonator cells \\
$\mathbf{8}$ & Gain & Gain of antenna \\
$\mathbf{9}$ & VSWR & Antenna's voltage standing wave ration \\
$\mathbf{1 0}$ & Bandwidth & Antenna's bandwidth \\
$\mathbf{1 1}$ & S11 & Return loss \\
\hline
\end{tabular}

Tab. 2 provides a statistical description of the dataset. Summary of the distribution, central tendency, and dispersion is presented in Tab. 2. Fig. 5 shows the correlation matrix of the dataset. As can be seen in the figure, $\mathrm{Xa}$ and Ya are highly correlated with the bandwidth. Then, Tm and $\mathrm{Wm}$ are slightly correlated with the bandwidth. The histogram for each feature is presented in Fig. 6. From this figure, the distribution of each feature can be studied and analyzed. Moreover, a Principal Component Analysis (PCA) has been performed on the dataset to study the responsi- 
bility of features for the variance. PCA showed that Ya is responsible for $17.21 \%$ of the variance while $\mathrm{W} 0 \mathrm{~m}$ is responsible for $15.3 \%$ of the variance. Distance between rings is responsible for $13.21 \%$ of the variance in the Metamaterial Antenna dataset.

In order to prepare data for the machine learning model, some preprocessing tasks have been applied. First, the null values in the bandwidth values have been handled by taking the average of the non-missing values of previous and next values of the missing value. In machine learning, data value ranges may affect the learning process and as a result the performance of the model will be impacted. For instance, KNN used Euclidean distance to measure the distance between data points. Features with higher bound will dominate and affect the calculation process. Therefore, it is an essential process to scale and normalize data to guarantee that all features lay in the same bounds and will be treated similarly by the machine learning model. One of the simple ways to scale data is the min-max scaler in which data features are scaled and bounded between the range of 0 and 1 using the min-max scaler. The following equation is used by the min-max scaler to perform its task.

Table 2: Description statistics of metamaterial dataset

\begin{tabular}{llllll}
\hline Feature & Wm & W0m & $\mathrm{dm}$ & $\mathrm{tm}$ & Xa \\
\hline Count & 572.0000 & 572.0000 & 572.000 & 572.0000 & 572.00000 \\
Mean & 2244.0482 & 400.5941 & 275.4257 & 224.4048 & 4063.2463 \\
Std & 691.5788 & 184.9052 & 150.901130 & 69.157890 & 3287.8620 \\
Min & 2142.9000 & 162.8600 & 77.1430 & 214.2900 & 0.000 \\
$\mathbf{2 5 \%}$ & 2142.9000 & 162.8600 & 77.14300 & 214.2900 & 1132.8000 \\
$\mathbf{5 0} \%$ & 2142.9000 & 325.7100 & 214.2900 & 214.2900 & 3543.5000 \\
$\mathbf{7 5 \%}$ & 2142.9000 & 488.5700 & 351.4300 & 214.2900 & 5954.3000 \\
Max & 6964.3000 & 651.4300 & 488.5700 & 696.4300 & 10776.000 \\
\hline Feature & Ya & Gain & vswr & Bandwidth & S11 \\
\hline Count & 572.000000 & 572.0000 & 572.0000 & 572.0000 & 572.0000 \\
Mean & 6947.4697 & 2.6785 & 2.09492 & 117.8989 & -16.1049 \\
Std & 5136.193313 & 0.683242 & 1.914750 & 11.233272 & 7.897142 \\
Min & 2142.9000 & -5.6543 & 1.041183 & 32.7599 & -33.9031 \\
$\mathbf{2 5} \%$ & 2142.9000 & 2.815006 & 1.187911 & 116.9174 & -21.3215 \\
$\mathbf{5 0} \%$ & 6964.3000 & 2.876220 & 1.438023 & 122.2199 & -14.9108 \\
$\mathbf{7 5 \%}$ & 11786.000 & 2.921877 & 1.725260 & 123.0597 & -11.4982 \\
Max & 16607.000 & 3.238539 & 8.377999 & 124.7401 & -2.08343 \\
\hline
\end{tabular}

Table 3: MSE results for base models and ensemble models

\begin{tabular}{lllll}
\hline DT & KNN & SVM & Average ensemble & Weight average ensemble \\
\hline 0.014926 & 0.015987 & 0.015529 & 0.014368 & 0.014080 \\
\hline
\end{tabular}




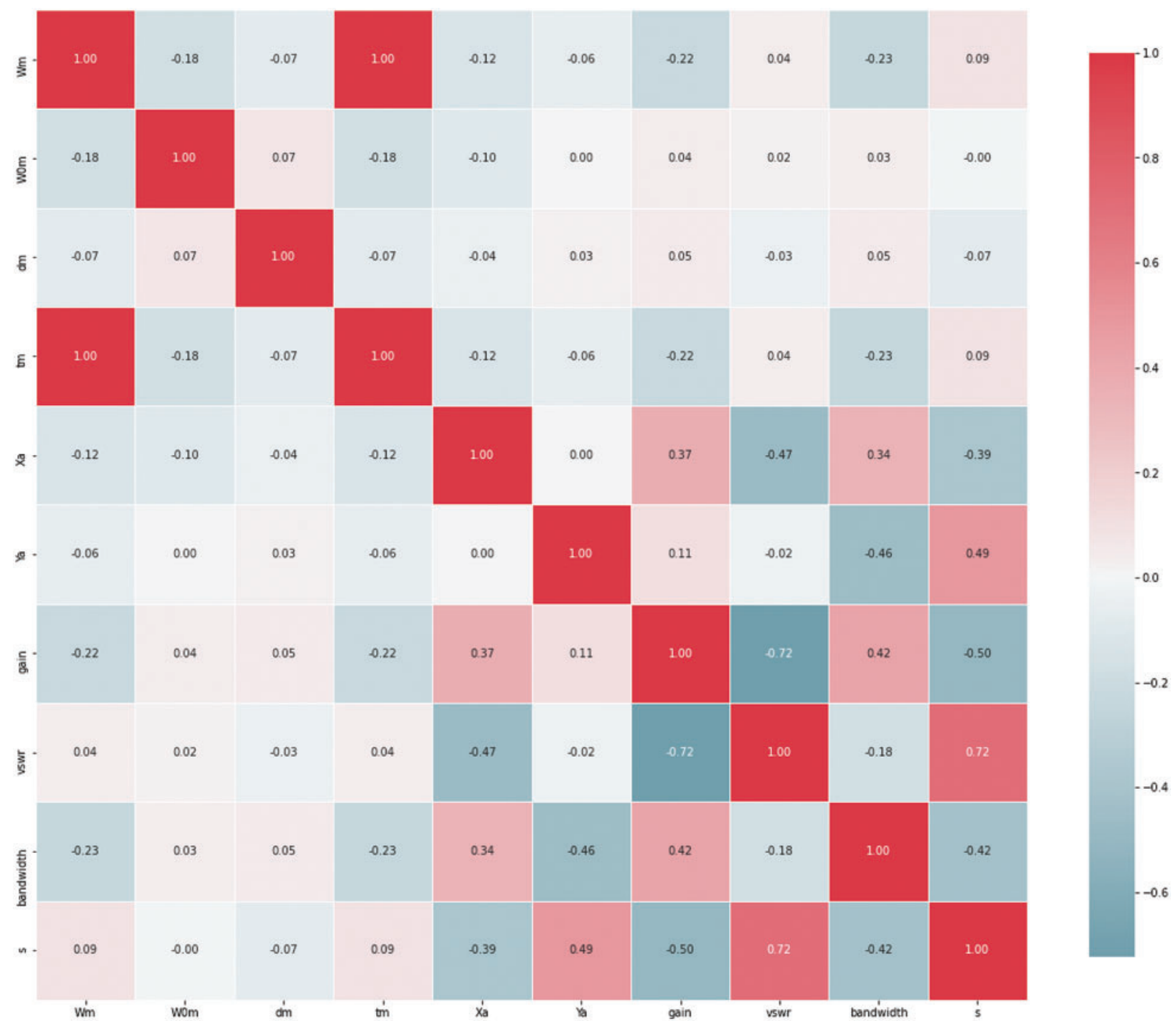

Figure 5: Correlation matrix of metamaterial antenna dataset

$X_{\text {scaled }}=\frac{X_{\text {val }}-X_{\text {min }}}{X_{\text {max }}-X_{\text {min }}}$

\subsection{Weight Average Ensemble Model}

The proposed weighted average ensemble model is based on optimize the weights for base models and then calculate the average ensemble on the weighted results. The Dynamic GroupBased Cooperative Optimizer (DGCO) algorithm is used to get the optimal weights for the ensemble model. As shown in Fig. 3, the DGCO algorithm optimize weights of three base models named Decision Tree (DT), K-Nearest Neighbor (KNN) and Support Vector Machines (SVM). 
W1 indicated the optimized weight of the DT model, W2 represents the optimized weight of the KNN model, and W3 is the optimized weight of the SVM model. After calculating the optimized weights of the three base models, the average ensemble is then calculated to get the final output result. The initial parameters of the DGCO algorithm are the population is set to 20 agents, maximum number of iterations equals 20, and number of runs are 20 for the tested dataset.

\section{Results and Discussion}

The Mean Square Error (MSE) is employed in this work as the main performance metric. The MSE metric can be calculated as follow to assess the performance.

$M S E=\frac{1}{n} \sum_{i=1}^{n}\left(Y_{i}-Y_{i}^{*}\right)^{2}$

where $Y_{i}$ represents a predicted value and $Y_{i}^{*}$ indicates the actual measured value. The total number of values is indicated as $n$ parameter.

The prediction of regression by the proposed weighted average ensemble model and the original values are shown in Fig. 7. The figure shows that the proposed model results are almost fitted to the original values. The proposed model is compared with three based models of the decision tree, K-nearest neighbor, and support vector machine models, in addition to, the average ensemble model as shown in Tab. 3. The results show that the proposed weight average ensemble model can achieve an MSE of (0.014080) which is better than other base and average ensemble models. This confirms that the proposed model can predict antenna bandwidth efficiently.
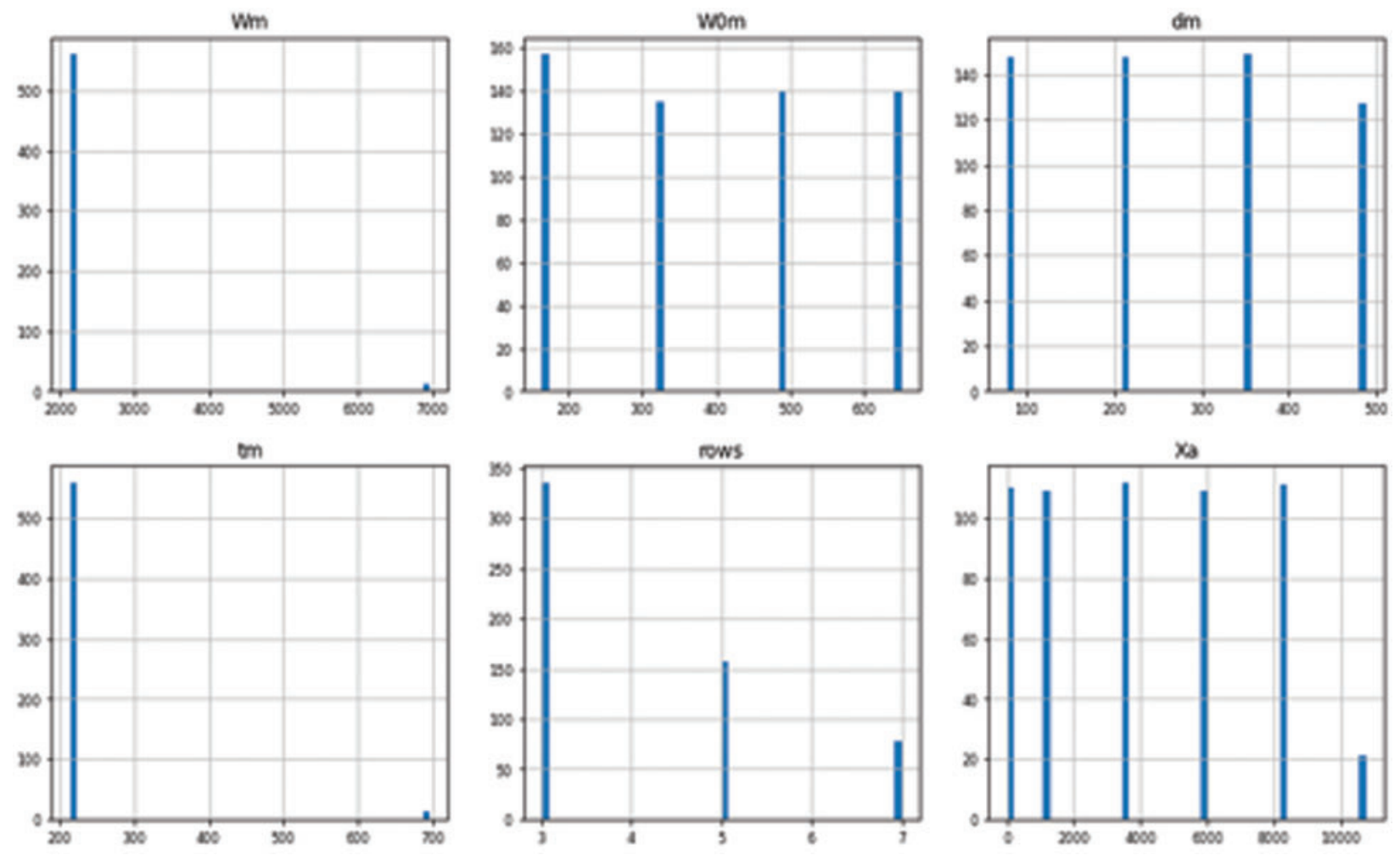

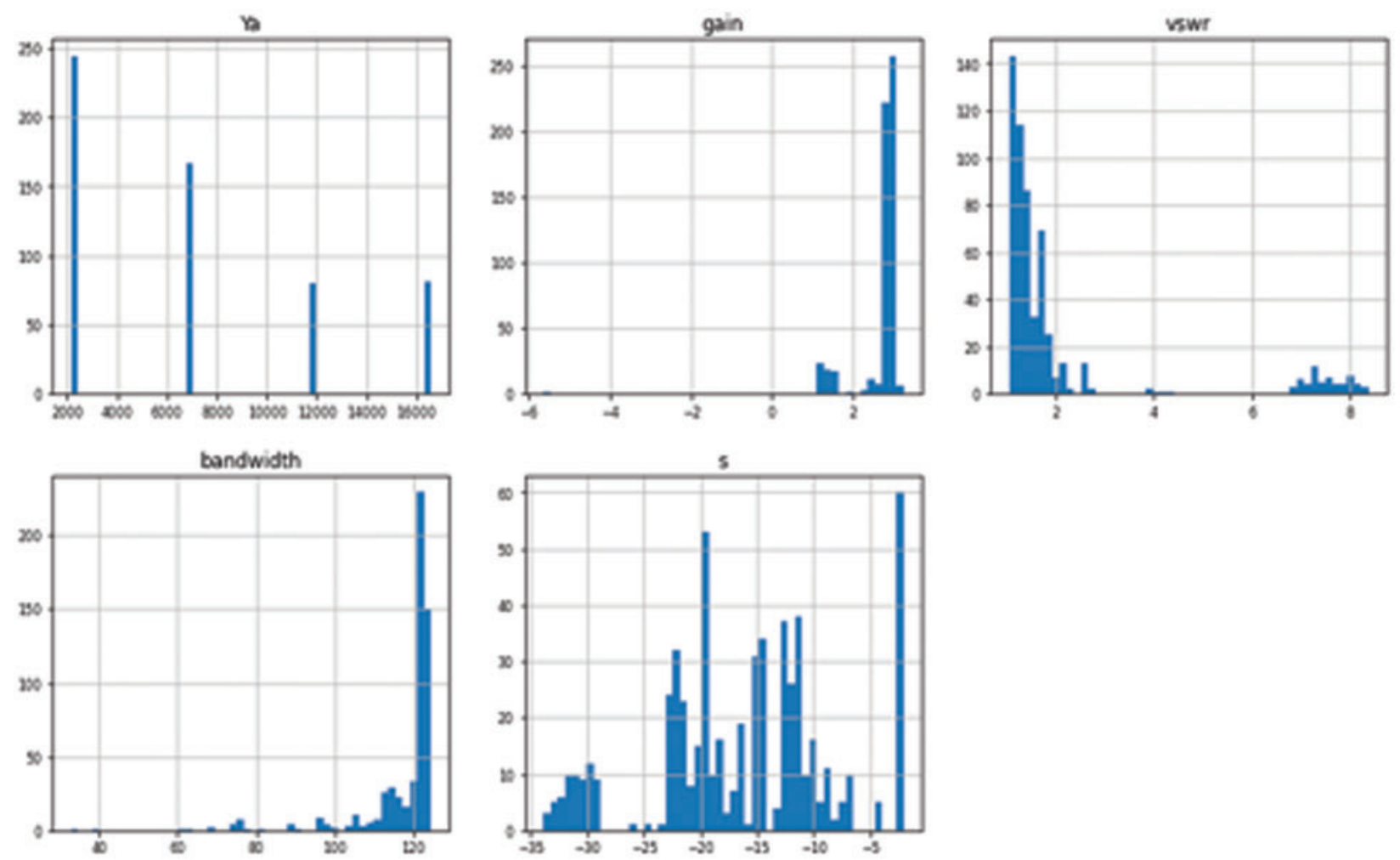

Figure 6: Distribution of dataset features (histogram)

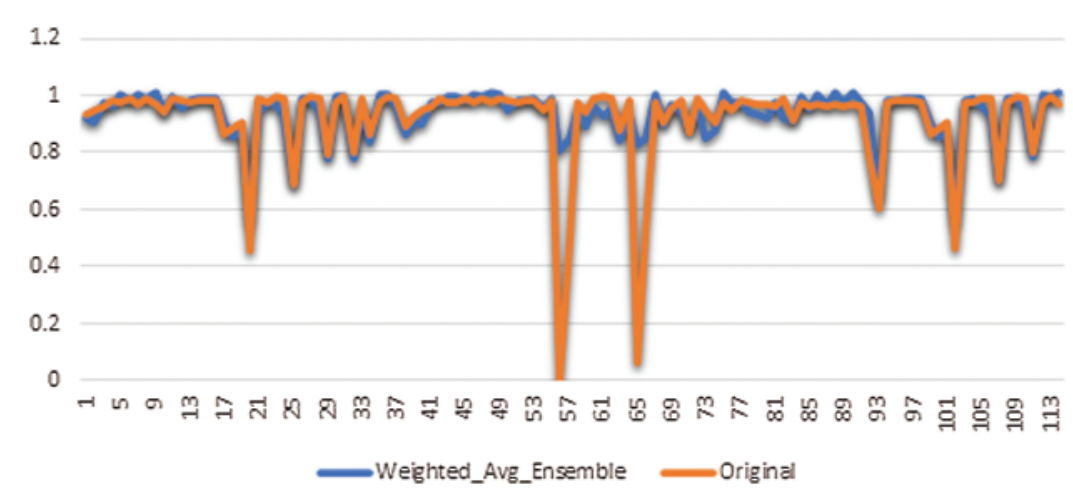

Figure 7: Original and estimated values

Fig. 8 shows the predicted and the actual residual values using residual, homoscedasticity, and QQ plots. Different plots confirm the performance of the proposed weighted average ensemble model to predict antenna bandwidth efficiently. The heat map is also represented in Fig. 8. The heat map is also confirming the stability of the proposed model. Tab. 4 shows a one-way analysis of variance (ANOVA) test results. ANOVA test is applied in this work for the measurement of the statistical differences between the proposed weighted average ensemble model and other models. Two hypotheses are formulated in this test. First, the null hypothesis $\left(H_{0}: \mu_{A}=\mu_{B}=\mu_{C}=\mu_{D}=\right.$ $\mu_{E}$ ), where $A$ : Proposed weighted average ensemble model, $B$ : Average ensemble model, $C$ : DT model, $D$ : KNN model, and $E$ : SVM model. The second hypothesis ( $H_{1}: \mu$ is not equal for all). 
The second hypothesis $H_{1}$ based on the results of the test is preferred. The results explain the superiority of the proposed weight average ensemble model and indicate the statistical significance of the algorithm.
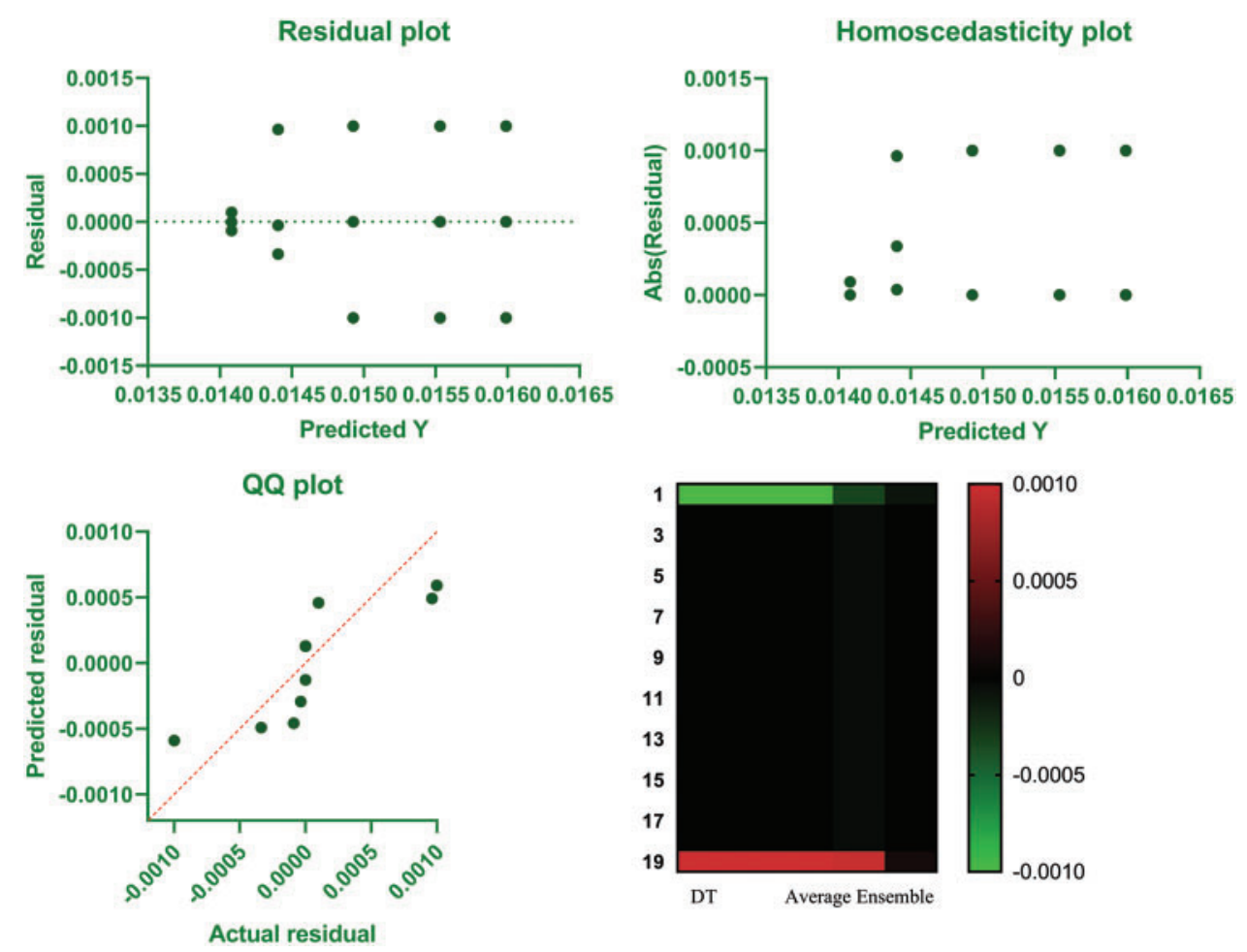

Figure 8: Residual, homoscedasticity, and QQ plots of the predicted and actual values

Table 4: ANOVA test for base models and ensemble models

\begin{tabular}{llllll}
\hline & SS & DF & MS & F (DFn, DFd) & P value \\
\hline Treatment (between columns) & $4.67 \mathrm{E}-05$ & 4 & $1.17 \mathrm{E}-05$ & $\mathrm{~F}(4,90)=148.4$ & $\mathrm{P}<0.0001$ \\
Residual (within columns) & $7.08 \mathrm{E}-06$ & 90 & $7.87 \mathrm{E}-08$ & - & - \\
Total & $5.38 \mathrm{E}-05$ & 94 & - & - & - \\
\hline
\end{tabular}

\section{Conclusion}

In this paper, a weighted average ensemble model is proposed to predict the bandwidth of the Metamaterial Antenna. To calculate the weights for base models, an optimization algorithm is used to find the optimal weights of the ensemble. Dynamic Group-Based Cooperative Optimizer (DGCO) is employed to search for optimal weight for the base models. The proposed model is compared with three based models and the average ensemble model. The results show that the proposed model is better than other models and can predict antenna bandwidth efficiently. The predicted and the actual residual values using residual, homoscedasticity, and QQ plots confirm 
the performance of the proposed weighted average ensemble model to predict antenna bandwidth efficiently. ANOVA test confirms the statistical differences between the proposed weighted average ensemble model and other models. In the future work, justification of the proposed model results compared to the EM simulation based on the optimized design parameters from other models as well as the proposed model will be considered. The proposed model will also be compared to other antenna optimization approaches such as antenna optimization using particle swarm optimization algorithm and genetic algorithm.

Funding Statement: The authors received no specific funding for this study.

Conflicts of Interest: The authors declare that they have no conflicts of interest to report regarding the present study.

\section{References}

[1] J. Suganthi, T. Kavitha and V. Ravindra, "Survey on metamaterial antennas," IOP Conference Series: Materials Science and Engineering, vol. 1070, no. 1, pp. 12086, 2021.

[2] M. Alibakhshikenari, B. S. Virdee, L. Azpilicueta, M. Naser-Moghadasi, M. O. Akinsolu et al., "A Comprehensive survey of metamaterial transmission-line based antennas: Design, challenges, and applications," IEEE Access, vol. 8, pp. 144778-144808, 2020.

[3] H. M. E. Misilmani and T. Naous, "Machine learning in antenna design: An overview on machine learning concept and algorithms," in 2019 Int. Conf. on High Performance Computing \& Simulation (HPCS), Dublin, Ireland, pp. 600-607, 2019.

[4] W. Naktong, A. Ruengwaree, N. Fhafhiem and P. Krachodnok, "Resonator rectenna design based on metamaterials for low-RF energy harvesting," Computers, Materials \& Continua, vol. 68, no. 2, pp. 1731$1750,2021$.

[5] N. I. Landy, S. Sajuyigbe, J. J. Mock, D. R. Smith, W. J. Padilla et al., "Perfect metamaterial absorber," Physical Review Letters, vol. 100, no. 20, pp. 207402, 2008.

[6] N. Kundtz and D. R. Smith, "Extreme-angle broadband metamaterial lens," Nature materials, vol. 9, no. 2, pp. 129-132, 2010.

[7] K. Sun, R. Fan, X. Zhang, Z. Zhang, Z. Shi et al., "An overview of metamaterials and their achievements in wireless power transfer," Journal of Materials Chemistry C, vol. 6, no. 12, pp. 2925-2943, 2018.

[8] X. Chen and W. Fan, "Ultrasensitive terahertz metamaterial sensor based on spoof surface plasmon," Scientific Reports, vol. 7, no. 1, pp. 1-8, 2017.

[9] Y. Dong and T. Itoh, "Metamaterial-based antennas," Proceedings of the IEEE, vol. 100, no. 7, pp. 2271-2285, 2012.

[10] J. Volakis, C. C. Chen and K. Fujimoto, Small Antennas: MIniaturization Techniques \& Applications. New York: McGraw Hill Professional, 2009.

[11] G. Geetharamani and T. Aathmanesan, "Design of metamaterial antenna for $2.4 \mathrm{GHz}$ WiFi applications," Wireless Personal Communications, vol. 113, no. 4, pp. 2289-2300, 2020.

[12] M. I. Saputra, S. R. Sulistiyanti, S. Purwiyanti and U. Murdika, "Design of prototype measuring motor vehicles velocity using hall effect sensor series A-1302 based on arduino mega 2560," in 2020 2nd Int. Conf. on Industrial Electrical and Electronics (ICIEE), Lombok, Indonesia, pp. 66-69, 2020.

[13] M. I. Jordan and T. M. Mitchell, "Machine learning: Trends, perspectives, and prospects," Science, vol. 349, no. 6245, pp. 255-260, 2015.

[14] A. Ibrahim, S. Tominaga and T. Horiuchi, "Spectral imaging method for material classification and inspection of printed circuit boards," Optical Engineering, vol. 49, no. 5, pp. 57201, 2010.

[15] R. Al-Hajj, A. Assi and M. M. Fouad, "Stacking-based ensemble of support vector regressors for one-day ahead solar irradiance prediction," in 2019 8th Int. Conf. on Renewable Energy Research and Applications (ICRERA), Brasov, Romania, pp. 428-433, 2019. 
[16] E. S. M. El-Kenawy, A. Ibrahim, S. Mirjalili, M. M. Eid and S. E. Hussein, "Novel feature selection and voting classifier algorithms for COVID-19 classification in CT images," IEEE Access, vol. 8, pp. 179317-179335, 2020.

[17] A. I. Károly, P. Galambos, J. Kuti and I. J. Rudas, "Deep learning in robotics: Survey on model structures and training strategies," IEEE Transactions on Systems, Man, and Cybernetics: Systems, vol. 51, no. 1, pp. 266-279, 2021.

[18] B. Yegnanarayana, Artificial Neural Networks. Delhi, India: PHI Learning Pvt. Ltd, 2009.

[19] W. S. Noble, "What is a support vector machine?" Nature biotechnology, vol. 24, no. 12, pp. 1565-1567, 2006.

[20] A. J. Myles, R. N. Feudale, Y. Liu, N. A. Woody and S. D. Brown, "An introduction to decision tree modeling," Journal of Chemometrics: A Journal of the Chemometrics Society, vol. 18, no. 6, pp. 275-285, 2004.

[21] N. Bhatia, "Survey of nearest neighbor techniques," International Journal of Computer Science and Information Security, vol. 8, no. 2, pp. 302-305, 2010.

[22] M. M. Fouad, A. I. El-Desouky, R. Al-Hajj and E.-S. M. El-Kenawy, "Dynamic group-based cooperative optimization algorithm," IEEE Access, vol. 8, pp. 148378-148403, 2020.

[23] M. SvenSSon and S. JoakiM, "Machine-learning technologies in telecommunications," Ericsson Review, vol. 3, pp. 29-33, 2008.

[24] T. Gaber, A. Tharwat, A. Ibrahim, V. Snáel and A. E. Hassanien, "Human Thermal face recognition based on random linear oracle (RLO) ensembles," in 2015 Int. Conf. on Intelligent Networking and Collaborative Systems, Taipei, Taiwan, pp. 91-98, 2015.

[25] E. S. M. El-Kenawy, A. Ibrahim, S. Mirjalili, M. M. Eid and S. E. Hussein, "Novel feature selection and voting classifier algorithms for COVID-19 classification in CT Images," IEEE Access, vol. 8, pp. 179317-179335, 2020.

[26] R. Al-Hajj, A. Assi and M. M. Fouad, "A predictive evaluation of global solar radiation using recurrent neural models and weather data," in 2017 IEEE 6th Int. Conf. on Renewable Energy Research and Applications (ICRERA), San Diego, CA, USA, pp. 195-199, 2017.

[27] M. Amrollahi, S. Hadayeghparast, H. Karimipour, F. Derakhshan and G. Srivastava, "Enhancing network security via machine learning: Opportunities and challenges," in Handbook of Big Data Privacy, Springer Nature Switzerland: Springer, Cham, pp. 165-189, 2020.

[28] A. H. El Zooghby, C. G. Christodoulou and M. Georgiopoulos, "A neural network-based smart antenna for multiple source tracking," IEEE Transactions on Antennas and Propagation, vol. 48, no. 5, pp. 768-776, 2000.

[29] U. Ozkaya and L. Seyfi, "Dimension optimization of microstrip patch antenna in $\mathrm{X} / \mathrm{Ku}$ band via artificial neural network," Procedia-Social and Behavioral Sciences, vol. 195, pp. 2520-2526, 2015.

[30] L. H. Manh, F. Grimaccia, M. Mussetta and R. E. Zich, "Optimization of a dual ring antenna by means of artifcial neural network," Progress In Electromagnetics Research B, vol. 58, pp. 59-69, 2014.

[31] Y. Kim, S. Keely, J. Ghosh and H. Ling, "Application of artificial neural networks to broadband antenna design based on a parametric frequency model," IEEE Transactions on Antennas and Propagation, vol. 55, no. 3, pp. 669-674, 2007.

[32] J. Nayak, B. Naik and H. Behera, "A comprehensive survey on support vector machine in data mining tasks: Applications \& challenges," International Journal of Database Theory and Application, vol. 8, no. 1, pp. 169-186, 2015.

[33] H. Lin, W. Y. Shin and J. Joung, "Support vector machine-based transmit antenna allocation for multiuser communication systems," Entropy, vol. 21, no. 5, pp. 471, 2019.

[34] S. Ülker, "Support vector regression analysis for the design of feed in a rectangular patch antenna," in 2019 3rd Int. Symp. on Multidisciplinary Studies and Innovative Technologies (ISMSIT), Ankara, Turkey, IEEE, pp. 1-3, 2019.

[35] R. Al-Hajj, A. Assi and M. Fouad, "Short-term prediction of global solar radiation energy using weather data and machine learning ensembles: A comparative study," Journal of Solar Energy Engineering, vol. 143, no. 5, pp. 51003, 2021. 
[36] B. S. Dhaliwal and S. S. Pattnaik, "Development of PSO-ANN ensemble hybrid algorithm and its application in compact crown circular fractal patch antenna design," Wireless Personal Communications, vol. 96, no. 1, pp. 135-152, 2017.

[37] S. Pattnaik, S. S. Pattnaik and B. S. Dhaliwal, "Modeling of circular fractal antenna using BFOPSO-based selective ANN ensemble," International Journal of Numerical Modelling: Electronic Networks, Devices and Fields, vol. 32, no. 3, pp. e2549, 2019.

[38] R. Machado, Metamaterial Antennas. 2019. [Online]. Available: https://www.kaggle.com/renanmav/meta material-antennas. [Accessed: 2021-07-17].

[39] N. Kurniawati, D. N. N. Putri and Y. K. Ningsih, "Random forest regression for predicting metamaterial antenna parameters," in 2nd Int. Conf. on Industrial Electrical and Electronics (ICIEE), Lombok, Indonesia, pp. 174-178, 2020. 